\title{
The impact of superstructures in the Cosmic Microwave Background
}

\author{
Stéphane Ilić ${ }^{1}$, Mathieu Langer ${ }^{2}$ and Marian Douspis ${ }^{2}$ \\ ${ }^{1}$ Institut de Recherche en Astrophysique et Planétologie, \\ 14 avenue Édouard Belin, 31400 Toulouse, France \\ email: stephane.ilic@irap.omp.eu \\ ${ }^{2}$ Institut d'Astrophysique Spatiale, Université Paris-Sud, \\ Bâtiment 121, 91405 Orsay Cedex, France \\ emails: mathieu.langer@ias.u-psud.fr, marian.douspis@ias.u-psud.fr
}

\begin{abstract}
In 2008, Granett et al. claimed a direct detection of the integrated Sachs- Wolfe (iSW) effect by a stacking approach of patches of the CMB, at the positions of identified superstructures. However, the high amplitude of their measured signal seems to be at odds with predictions from the standard model of cosmology. However, multiple questions arise from these results and their expected value : I propose here an original theoretical prediction of the iSW effect produced by such superstructures. I use simulations based on GR and the LTB metric to reproduce cosmic structures and predict their exact full theoretical iSW effect. Expected amplitudes are consistent with the measured signal ; however the latter shows non-reproducible features that are hardly compatible with $\Lambda$ CDM predictions.
\end{abstract}

Keywords. cosmic microwave background, large-scale structure of universe, dark energy, general relativity

\section{Introduction}

Dark Energy (DE) is one of the great mysteries of modern cosmology. The integrated Sachs-Wolfe effect (iSW) is an original probe of DE, linked to the large-scale structure of the Universe and the cosmic microwave background (CMB). Indeed, the accelerated expansion stretches gravitational potentials, therefore changing the frequency of the CMB photons that cross them. This effect is integrated along the whole path of the photons and shift the temperature of CMB photons by an amount defined by :

$$
\delta_{T}^{\mathrm{iSW}}=2 \int \dot{\Phi} d t \quad(\Phi=\text { grav.potential })
$$

Consequently, the iSW effect has a direct but very weak impact on the largest scales of the power spectrum of the CMB temperature fluctuations. It therefore requires the use of external data to be detectable. The conventional approach is to correlate the CMB with a tracer of the matter distribution - usually galaxy surveys. Although attempted numerous times (see Dupé et al., 2011 for a review), these have yet to give a definitive and unambiguous detection of the iSW effect. This is mainly due to the shortcomings of current surveys, not deep enough and/or with too small a sky coverage.

In this unclear context, another approach would be to focus on the individual objects expected to leave the biggest imprint on the CMB, i.e. the largest superstructures in the Universe. While their individual imprint is drowned in the primordial CMB fluctuations, we can average patches of the $\mathrm{CMB}$ at the locations of many superstructures in order to cancel the random CMB fluctuations while enhancing the iSW signal. In their pioneering work, Granett et al. (2008) claimed a $4 \sigma$ detection of the iSW effect by stacking CMB 


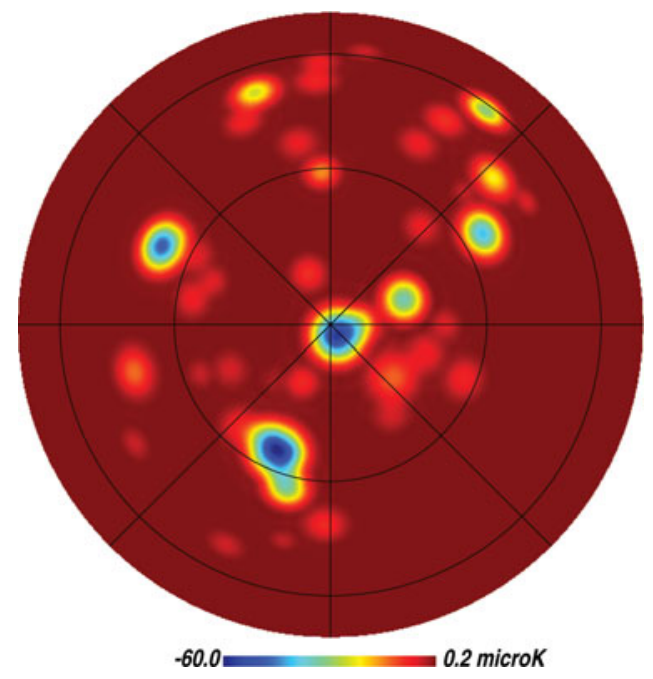

Figure 1. Map of the theoretical temperature shift due to the 50 voids of Granett et al. 2008. The map is centred on the galactic north pole.

patches at the positions of 100 superstructures, identified in the SDSS. However, the measured amplitude was reported to be at odds with $\Lambda$ CDM predictions (e.g. Cai et al., 2013), while some peculiar features were also noted in the signal (Ilić et al., 2013) such as the large hot ring around the cold signal from voids.

\section{Simulating a superstructure and its impact on the CMB}

The results of the stacking approach raised a number of questions concerning the nature of the expected signal. This led me to propose an original theoretical prediction of the iSW effect produced by superstructures : I modelled each structure and its evolution using the Lemaitre-Tolman-Bondi (LTB) metric of GR, the most general metric with a spherical symmetry. Using the data from the Granett et al. catalogue, I focused on reproducing their 50 voids and their evolution, and then predict the exact full theoretical iSW effect of these structures by solving geodesic equations for crossing photons.

As expected, the mean effect of these voids is indeed small (about $-10 \mu \mathrm{K}$ for photons going through their center). However, using my simulations I showed that in theory, only a fifth of the 50 voids of Granett (as intuited, the largest ones) should contribute for the majority of the predicted signal. However, removing these voids from the real CMB stacking shows that they have almost no contribution to the measured signal. This finding may cast some doubts on whether the measured signal is really due to the iSW effect of the voids.

Going further, I was also able to reconstruct the theoretical "iSW map" associated with these 50 voids (see Fig. 1), i.e. the temperature shift due to the iSW effect associated to each point in the sky, accounting for all the voids present on the corresponding line of sight. Similarly to the analysis on real data, I can perform the stacking procedure on this iSW map, and obtain the expected signal from these voids. However, one should also account for the effect of the CMB primordial fluctuations in this measurement. To do so, I generated many Gaussian realisations of the CMB (with the current best-fit cosmological parameters) that I added on top of (or rather, under) these secondary iSW fluctuations. In the end, I was able to reconstruct both the expected temperature profile 


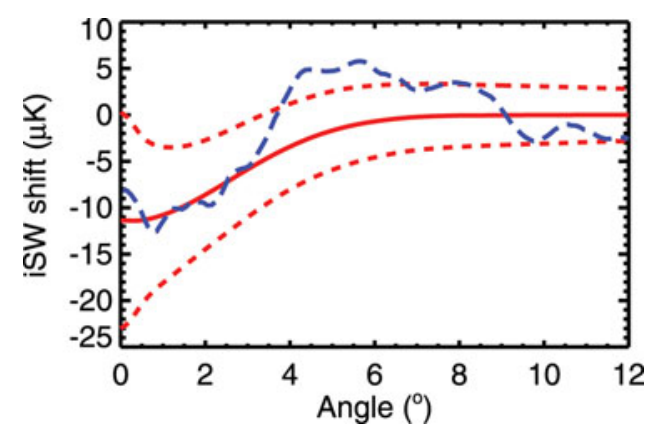

Figure 2. Theoretical iSW temperature profile (solid red) and $1 \sigma$ limits for the contamination due to primordial CMB fluctuations. The measured temperature profile of the "real" stacked CMB patches is shown in blue.

of the resulting stacked image, but also and foremost its $1 \sigma$ limits due to CMB noise (see Fig. 2).

The final step in this analysis was to compare those predictions with the measure temperature profile coming from the stacking of real CMB patches (shown also in Fig. 2). Contrary to previous claims in the literature, I show here that the measured signal is compatible with (my) $\Lambda$ CDM predictions at the $\sim 1 \sigma$ level. The high reported amplitude seems to be merely due to random (and not particularly rare) fluctuations that artificially enhanced the signal. It is therefore compatible with the expected iSW from such structures.

\section{References}

Cai, Y.-C., Neyrinck, M. C., Szapudi, I., Cole, S., \& Frenk, C. S. 2014, ApJ, 786, 110

Dupé, F.-X., Rassat, A., Starck, J.-L., \& Fadili, M. J. 2011, A\&3A, 534, A51

Granett, B. R., Neyrinck, M. C., \& Szapudi, I. 2008, ApJ, 683, L99-L102

Ilić, S., Langer, M., \& Douspis, M. 2013, A\& A, 556, A51 